\title{
The MAPK pathway is involved in the regulation of rapid pacing-induced ionic channel remodeling in rat atrial myocytes
}

\author{
WEI CHENG, YUN ZHU and HAIDONG WANG
}

Department of Cardiothoracic Surgery, Southwest Hospital, Third Military Medical University, Chongqing 400038, P.R. China

Received March 14, 2015; Accepted January 11, 2016

DOI: $10.3892 / \mathrm{mmr} .2016 .4862$

\begin{abstract}
Alterations to the expression L-type calcium channels (LTCCs) and Kv4.3 potassium channels form the possible basis of atrial electrical remodeling during rapid pacing. The mitogen-activated protein kinase (MAPK) pathway is affected by increases in cytoplasmic $\mathrm{Ca}^{2+}$, and therefore represents an attractive candidate for the regulation and mediation of $\mathrm{Ca}^{2+}$-induced ion channel remodeling. The present study aimed to investigate alterations to the ion channel-MAPK axis, and to determine its influence on ion channel remodeling during atrial fibrillation. Rat atrial myocytes were isolated, cultured, and in vitro rapid pacing was established. Intracellular $\mathrm{Ca}^{2+}$ signals were monitored using the Fluo-3/AM Ca ${ }^{2+}$ indicator. Verapamil, PD98058 and SB203580 were added to the culture medium of various groups at specific time-points. The mRNA expression levels of LTCC- $\alpha 1 \mathrm{c}$ and Kv4.3 potassium channels were detected by reverse transcription-polymerase chain reaction. Western blotting was performed to determine the expression levels of channel and signaling proteins. The results demonstrated that fast pacing significantly increased the intracellular $\mathrm{Ca}^{2+}$ concentration in atrial myocytes, whereas treatment with verapamil markedly inhibited this increase. In addition, verapamil significantly antagonized the rapid pacing-induced activation of extracellular signal-regulated kinase (ERK) and p38MAPK. These results indicated that the MAPK pathway may have an important role in the opening of LTCCs, and alterations to MAPK molecule expression could affect the expression and remodeling of ion channels.
\end{abstract}

\section{Introduction}

Atrial fibrillation (AF) is one of the most common types of arrhythmia encountered in clinical practice (1). The main characteristics of AF are the alterations in atrial

Correspondence to: Professor Haidong Wang, Department of Cardiothoracic Surgery, Southwest Hospital, Third Military Medical University, 30 Gaotanyan Street, Chongqing 400038, P.R. China E-mail: wanghdtmmu@163.com

Key words: rapid pacing, ion channel, mitogen-activated protein kinase electrophysiology and structure, which is termed electrophysiological and structural remodeling and is important in the development, maintenance and recurrence of $\operatorname{AF}(2,3)$. Ion influx via calcium and potassium channels is necessary for cellular neurochemical changes. Alterations to the expression of ion channels, particularly L-type calcium channels (LTCCs) and Kv4.3 potassium channels, form the possible basis of early remodeling during rapid pacing (4-6). However, the pathogenetic mechanisms underlying atrial structural remodeling remain poorly understood.

Alterations to cytoplasmic $\mathrm{Ca}^{2+}$ concentration have a critical role in the occurrence and maintenance of AF (7). Stimulation, such as the arrival of an action potential, increases $\mathrm{Ca}^{2+}$ entry from the extracellular space through LTCCs, and the resultant intracellular $\mathrm{Ca}^{2+}$ elevation (calcium overload) mediates atrial electrical remodeling (8). Increased cytoplasmic $\mathrm{Ca}^{2+}$ can not only exert feedback inhibition, which curtails further $\mathrm{Ca}^{2+}$ entry (9), but also activates other signaling molecules, including calmodulin and mitogen-activated protein kinase (MAPK) $(10,11)$. A previous study demonstrated that patients with permanent AF exhibit severe alterations in cardiac tissue architecture, accompanied by increased atrial expression of extracellular signal-regulated kinase 1/2 (ERK-1/2), one of the three major members of the MAPK family (12). In addition, L-type $\mathrm{Ca}_{\mathrm{v}} 1.2$ channels have previously been demonstrated to activate $\mathrm{Ca}^{2+}$-mediated second messenger pathways, including the MAPK pathway (13). These studies suggest that the MAPK pathway may consist of attractive candidate kinases for the regulation and mediation of $\mathrm{Ca}^{2+}$-induced ion channel remodeling. The present study aimed to investigate alterations to the ion channel-MAPK axis, and to determine its influence on ion channel remodeling during AF.

\section{Materials and methods}

Isolation and culture of rat atrial myocytes. The present study and all experimental protocols involved were approved by the Institutional Animal Care and Use Committee of the Third Military Medical University (Chongqing, China). A total of 20 female Wistar rats (2-week-old) were purchased from the Experimental Animal Center of the Third Military Medical University (Chongqing, China). All animals were housed in the same temperature $\left(22 \pm 2^{\circ} \mathrm{C}\right)$ and humidity-controlled holding facility with free access to food and water, and were exposed to a regular 12/12-h light/dark cycle. Rats were anesthetized with 
over-dosed $\mathrm{CO}_{2}$ (Chongqing Qunhe Medical Instrument Co., Ltd., Chongqing, China) and then fixed in a supine position. After sterilization, an incision was made along the right edge of the sternum and the chest wall was removed. The heart was retrieved and then washed in cold phosphate-buffered saline. Subsequently, the left and right atria were isolated and washed in serum-free Dulbecco's modified Eagle's medium (DMEM; Gibco; Thermo Fisher Scientific, Inc., Waltham, MA, USA). Under aseptic conditions, the right atrial appendage was trimmed with scissors into small sections of $\sim 1 \mathrm{~mm}^{3}$, which were digested with $0.08 \%$ trypsin at $37^{\circ} \mathrm{C}$ for $5 \mathrm{~min}$. The digestion was inactivated by the addition of medium containing $5 \%$ fetal bovine serum (Gibco; Thermo Fisher Scientific, Inc.). The solution was maintained at room temperature for $5 \mathrm{~min}$ and the supernatant was subsequently filtered through a 100-mesh filter. Digestion was performed twice. Finally, suspensions of single cells were prepared by treatment of the digested product with $0.1 \%$ type II collagenase (Sigma-Aldrich, Steinheim, Germany) at $37^{\circ} \mathrm{C}$ for $15 \mathrm{~min}$. The cells were then seeded into flasks at a density of $\sim 1 \times 10^{8} / 1$, followed by incubation with DMEM supplemented with $10 \%$ fetal bovine serum at $37^{\circ} \mathrm{C}$ in an atmosphere containing $5 \% \mathrm{CO}_{2}$. In the control group, after $72 \mathrm{~h}$ of routine culture, the medium was replaced with serum-free DMEM for $24 \mathrm{~h}$, and rapid pacing was performed for up to $24 \mathrm{~h}$. In the experimental groups, after $72 \mathrm{~h}$ of routine culture, the medium was replaced with serum-free DMEM for $24 \mathrm{~h}$, and the following reagents were added to the media of the various groups: Verapamil (Sigma-Aldrich, St. Louis, MO, USA), PD98058 (Sigma-Aldrich), SB203580 (Sigma-Aldrich), or PD98058 + SB203580 at the same concentration $(10 \mu \mathrm{mol} / \mathrm{l})$ for $30 \mathrm{~min}$.

Rapid pacing of atrial myocardial cells. Once cell confluence reached $\sim 80 \%$, the culture dishes were placed in an electric field. The cells were stimulated with $10 \mathrm{~Hz}, 1.5 \mathrm{~V} / \mathrm{cm}$ using the BL-420E+ Biological and Functional Experimental system (PCLab, Chengdu, China) at 3, 6, 12 and $24 \mathrm{~h}$.

Monitoring of intracellular $\mathrm{Ca}^{2+}$. The intracellular $\mathrm{Ca}^{2+}$ signals were recorded in the rat atrial myocytes using the Fluo-3/AM $\mathrm{Ca}^{2+}$ indicator (Invitrogen; Thermo Fisher Scientific, Inc.) at a concentration of $2 \mu \mathrm{mol} / 1$. Briefly, the growth medium was removed from the cells and was replaced with dye-loading medium (100 $\mu \mathrm{l} /$ well) containing $2 \mu \mathrm{M}$ Fluo-3/AM Ca ${ }^{2+}$ indicator and 20\% Pluronic acid F127 in Locke's buffer (154 mM $\mathrm{NaCl}, 5.6 \mathrm{mM} \mathrm{KCl}, 1.0 \mathrm{mM} \mathrm{MgCl}, 2.3 \mathrm{mM} \mathrm{CaCl}, 8.6 \mathrm{mM}$ HEPES, $5.6 \mathrm{mM}$ glucose and $0.1 \mathrm{~m} \mathrm{M}$ glycine; $\mathrm{pH}$ 7.4). The cells were incubated at $37^{\circ} \mathrm{C}$ in an atmosphere containing $5 \% \mathrm{CO}_{2}$ for $30 \mathrm{~min}$, and were washed four times with fresh DMEM. Calcium imaging was carried out using a Leica TCS SP5 confocal microscope (Leica Microsystems, Wetzlar, Germany).

Reverse transcription-polymerase chain reaction ( $R T-P C R)$ analysis. The mRNA expression levels of LTCC- $\alpha 1 \mathrm{c}$ and Kv4.3 potassium channels were detected by RT-PCR. Total cellular RNA was extracted from the rat atrial myocytes of each group using TRIzol ${ }^{\circledR}$ reagent (Invitrogen; Thermo Fisher Scientific, Inc.), and cDNA was prepared using a Superscript II First-Strand cDNA Synthesis kit (Invitrogen; Thermo
Fisher Scientific, Inc.), according to manufacturer's protocol. RT-PCR was performed and the primer sequences were designed as follows: $\alpha 1 \mathrm{c}$, forward 5'-ATGGAGGCTGGAGCC CAGATTGA-3', reverse 5'-GACATTGAGGTCCGCACC GAAGG-3' (annealing temperature, $61.3^{\circ} \mathrm{C}$ ); Kv4.3, forward 5'-GCAGCAACCTGAAATCTGAAACT-3', reverse 5'-GAT AAGCAATGAACCCATCTCCA-3' (annealing temperature, $56.1^{\circ} \mathrm{C}$ ); and $\beta$-actin, forward 5'-TGA GAGGGAAATCGT GCGTGAC-3' and reverse 5'-ATCTGCTGGAAGGTGGAC AGTGAG-3' (annealing temperature: $53.9^{\circ} \mathrm{C}$ ). These primers were synthesized by Sangon Biotech Co., Ltd. (Shanghai, China). PCR was run on the Veriti Thermal Cycler (Thermo Fisher Scientific, Inc.) with each sample at $20 \mu 1$, including $1 \mu \mathrm{l}$ primer, $10 \mu \mathrm{l}$ Taq Master mix (2X, E00019, Genscript Biotech Co., Nanjing, China) and $9 \mu \mathrm{l}$ deionized/distilled water. The amplification process was performed for 25 cycles: Initial denaturation at $94^{\circ} \mathrm{C}$ for $45 \mathrm{sec}$, annealing for $30 \mathrm{sec}$ at the indicated temperature, and final extension for $5 \mathrm{~min}$ at $72^{\circ} \mathrm{C}$. PCR products were separated by $1 \%$ agarose gel electrophoresis and were stained with ethidium bromide.

Western blot analysis. A total of $1.5 \times 10^{6}$ rat atrial myocytes from each group were lysed in $0.5 \mathrm{ml}$ radioimmunoprecipitation assay [50 mM Tris- $\mathrm{HCl}$ (pH 7.2), $150 \mathrm{mM} \mathrm{NaCl}, 1 \%$ NP40, 0.1\% SDS, 0.5\% DOC, $1 \mathrm{mM}$ PMSF, $25 \mathrm{mM} \mathrm{MgCl}$ and phosphatase inhibitor cocktail (Shanghai Qcbio Science $\&$ Technologies Co., Ltd., Shanghai, China)] buffer and $5 \mu 1$ phenylmethylsulfonyl fluoride. Cell homogenates were centrifuged at $12,000 \times \mathrm{g}$ for $30 \mathrm{~min}$ and the resulting supernatant (total tissue homogenate) was stored at $-80^{\circ} \mathrm{C}$ for further analysis. Protein concentration was quantified using a BCA Protein Quantification kit (Abcam, Cambridge, MA, USA) and a total of $15 \mu \mathrm{g}$ protein from each group was separated by $10 \%$ sodium dodecyl sulfate-polyacrylamide gel electrophoresis and transferred to polyvinylidene difluoride membranes (EMD Millipore, Temecula, CA, USA). The membranes were blocked by incubation with $5 \%$ BSA for $1 \mathrm{~h}$ and then incubated overnight at $4^{\circ} \mathrm{C}$ for $1 \mathrm{~h}$ with primary antibodies, followed by incubation with horseradish peroxidase (HRP)-conjugated secondary antibodies for $2 \mathrm{~h}$ at room temperature. The following primary antibodies were used: Rabbit polyclonal anti-rat ERK (cat. no. sc-94; 1:1,000; Santa Cruz Biotechnology, Inc., Dallas, TX, USA), rabbit polyclonal anti-rat phosphorylated (p)-ERK (cat. no. sc-16982; 1:500; Santa Cruz Biotechnology, Inc.), rabbit polyclonal anti-rat p38MAPK (cat. no. sc-535; 1:500; Santa Cruz Biotechnology, Inc.), rabbit anti-rat p-p38MAPK (1:500; cat. no. sc-101759; Santa Cruz Biotechnology, Inc.), rabbit anti-rat $\alpha 1 \mathrm{c}$ (cat. no. AB5150; 1:2,000; Chemicon; EMD Millipore, Billerica, MA, USA), rabbit anti-rat Kv4.3 (cat. no. AB5194; 1:1,000; Chemicon; EMD Millipore) and rabbit polyclonal anti-GAPDH antibody (cat. no. ABS16; EMD Millipore) was used as internal control. The secondary antibodies used were as follows: HRP-conjugated goat anti-rabbit immunoglobulin G (cat. no. ANT-178; 1:5,000 dilution; Beijing Dingguo Biotechnology Co., Ltd., Beijing, China). HRP chemiluminescent substrate was used and stained blots were visualized using an Odyssey Imaging System (Li-Cor Biosciences, Lincoln, NE, USA). Gel quantification was conducted using ImageJ software (version 1.2; National Institutes of Health, Bethesda, MD, USA). 

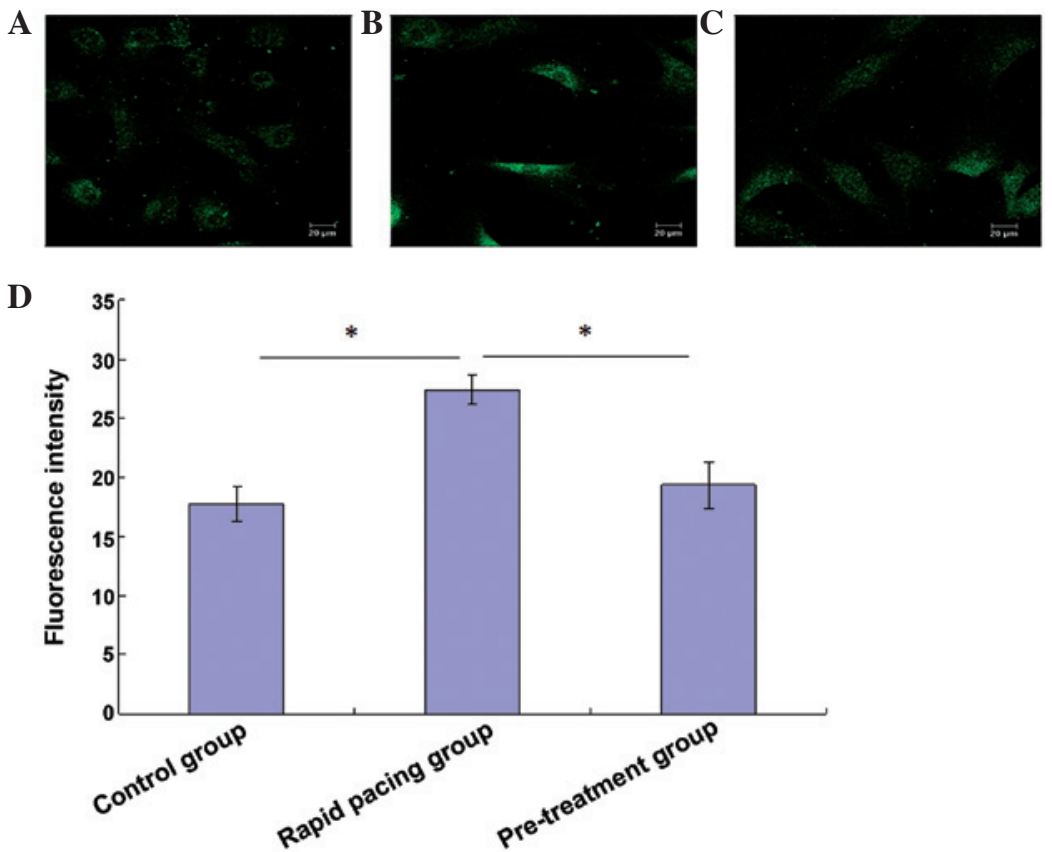

Figure 1. Alterations to intracellular $\mathrm{Ca}^{2+}$ concentration. Fluorescence imaging of (A) untreated cells; (B) cells that underwent fast pacing; and (C) cells that underwent fast pacing that were pretreated with verapamil. (D) Quantification of intracellular Ca ${ }^{2+}$ fluorescence intensity. Data are presented as the mean \pm standard deviation. ${ }^{*} \mathrm{P}<0.01$.

Statistical analysis. Statistical analysis was performed using SPSS version 17.0 (SPSS, Inc., Chicago, IL, USA). Group comparison was performed using one-factor analysis of variance. Data are presented as the mean \pm standard deviation. $\mathrm{P}<0.05$ was considered to indicate a statistically significant difference.

\section{Results}

Verapamil pretreatment inhibits fast pacing-induced increases in intracellular $\mathrm{Ca}^{2+}$ concentration. The Fluo-3/AM $\mathrm{Ca}^{2+}$ indicator was used to monitor $\mathrm{Ca}^{2+}$ concentration (Fig. 1). Fast pacing of rat atrial myocytes significantly increased intracellular $\mathrm{Ca}^{2+}$ concentrations, which was reflected by increases in intracellular calcium fluorescence intensity $24 \mathrm{~h}$ after rapid pacing (Fig. 1A, B and D). Conversely, verapamil pretreatment prior to pacing significantly reduced the increase in intracellular calcium fluorescence intensity (Fig. 1C and D).

Alterations to ERK and p38MAPK protein expression during fast pacing. The total and phosphorylated forms of two subtypes of the MAPK family: ERK and p38MAPK were detected by western blotting (Fig. 2). The expression levels of total and p-ERK protein were significantly increased $24 \mathrm{~h}$ after fast pacing (Fig. 2A and C). The expression levels of phosphorylated p38MAPK were upregulated $24 \mathrm{~h}$ after fast pacing; however, total p38MAPK expression remained changed (Fig. 2B and C). Notably, verapamil pretreatment significantly inhibited the upregulation of both phosphorylated proteins (Fig. 2A and C).

Effects of PD98059 on the expression of ion channel proteins. The mRNA expression levels of LTCC- $\alpha 1 \mathrm{c}$ and Kv4.3 potassium channels were significantly lower $24 \mathrm{~h}$ after fast pacing, as compared with those prior to pacing (Fig. 3A). This downregulation was diminished by pretreatment with the ERK1/2-specific inhibitor PD98059. However, compared with the untreated group, the expression levels of $\alpha 1 \mathrm{c}$ and $\mathrm{Kv} 4.3$ were lower in the PD98059 group. The results of western blotting indicated that the protein expression levels corroborated the PCR results (Fig. 3B).

Effects of SB203580 on the expression of ion channel proteins. Similar to the effects of PD98059, treatment with SB203580 could rescue the fast pacing-induced downregulation of $\alpha 1 \mathrm{c}$ and Kv4.3 expression (Fig. 3C and D). However, the effects were not as noticeable as the effects of PD98059.

Effects of PD98059 and SB203580 co-treatment on the expression of ion channel proteins. Simultaneous administration with PD98059 and SB203580 prior to fast pacing was able to inhibit the downregulation of $\alpha 1 \mathrm{c}$ and Kv4.3 expression (Fig. 3E and F). However, these reagents could not totally rescue the expression of $\alpha 1 \mathrm{c}$ and Kv4.3 to normal levels.

\section{Discussion}

Intracellular calcium overload has been reported as a key factor for electrical remodeling in AF (14). LTCCs, which are a member of the voltage-dependent calcium channel family, act as a voltage sensor for excitation-contraction coupling, and are a major pathway for calcium influx in cardiac and skeletal muscle (15). LTCCs function as macromolecular complexes, which are involved in the ubiquitous regulation of intracellular signaling (16). The $\mathrm{Ca}^{2+}$ influx through LTCCs forms the basis of the characteristic prolonged 'plateau' phase of the cardiac action potential (17). LTCC function is multifaceted, however in cardiac muscle cells it has an essential role in coupling 
A

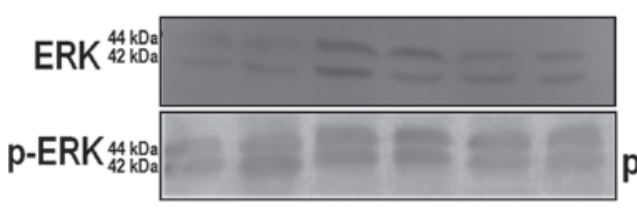

C

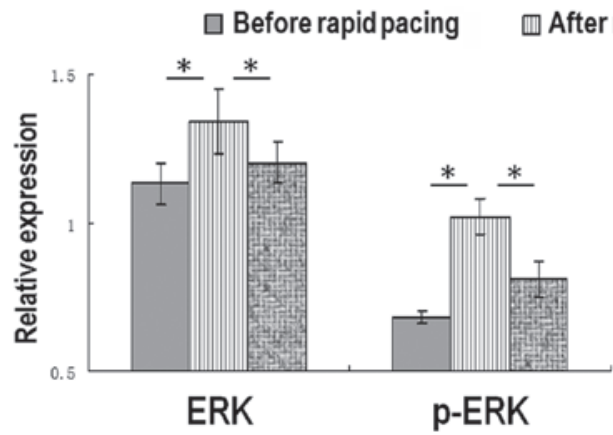

B

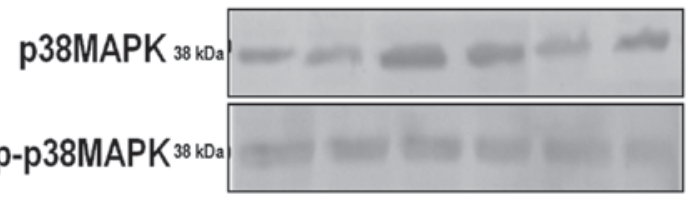

回 Pretreatment

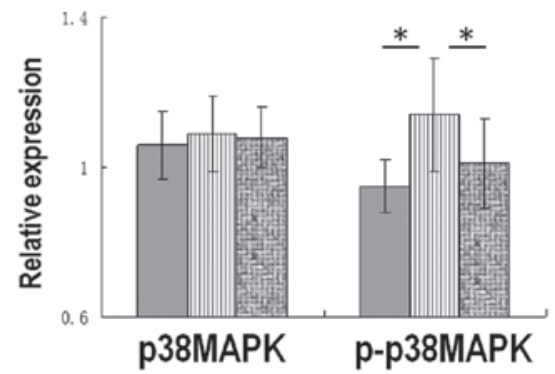

Figure 2. Protein expression levels of extracellular signal-regulated kinase (ERK) and p38 mitogen-activated protein kinase (MAPK), as detected by western blotting. (A) Expression of ERK and phosphorylated (p)-ERK, (B) p38MAPK and p-p38MAPK in the un-treated group (left 2 bands), fast pacing group (middle 2 bands) and fast pacing cells pretreated with verapamil (right 2 bands). (C) Quantification analysis. Data are presented as the mean \pm standard deviation. ${ }^{*} \mathrm{P}<0.01$.
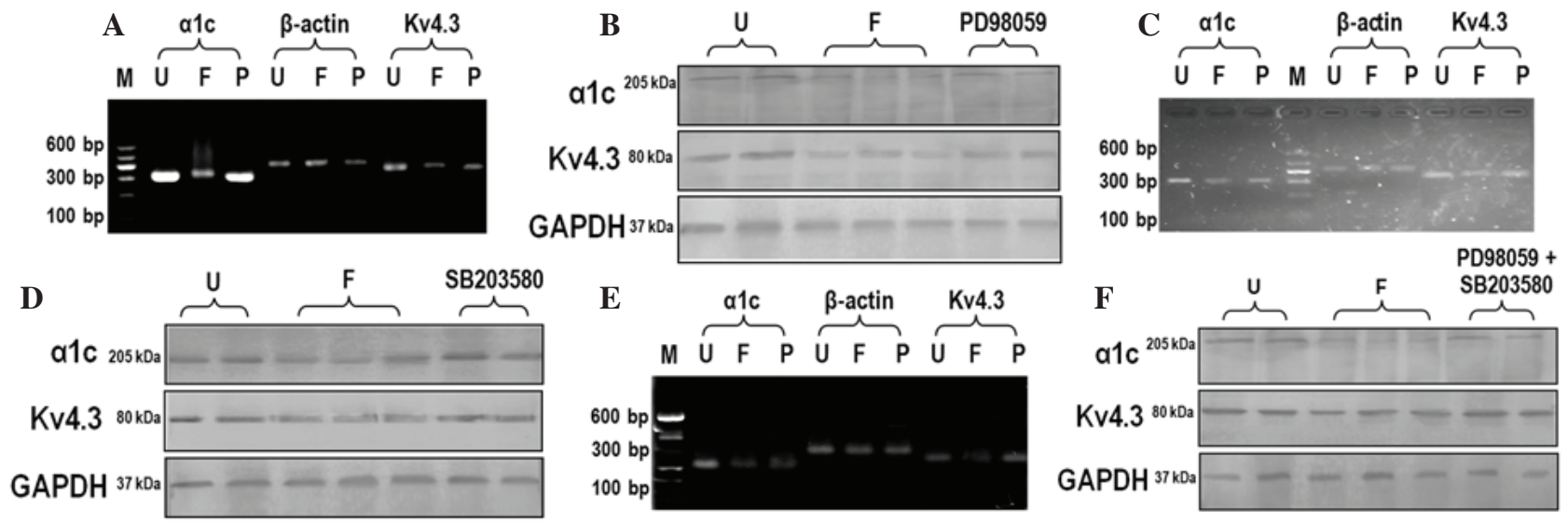

Figure 3. Effects of extracellular signal-regulated kinase (ERK) and p38 mitogen-activated protein kinase (MAPK) inhibition on the expression of ion channel proteins. (A and B) PD98059, (C and D) SB203580 and (E and F) a combination of these inhibitors were used to pretreat the cells. (A, C and E) Reverse transcription-polymerase chain reaction analysis of the mRNA expression of $\alpha 1 \mathrm{c}$ and Kv4.3. (B, D and F) Western blotting analysis of $\alpha 1 \mathrm{c}$ and Kv4.3 protein expression. M, marker; U, untreated group; F, fast pacing group; P, pretreated group; GAPDH, glyceraldehyde 3-phosphate dehydrogenase.

excitation to contraction. In cardiac myocytes, LTCCs increase $\mathrm{Ca}^{2+}$ entry and trigger calcium release from the sarcoplasmic reticulum by activating intracellular calcium channel ryanodine receptors (18). In addition, calcium is an important second messenger, which has a key role in calmodulin-dependent activation of signaling cascades that convey local calcium signals to the nucleus (19). Therefore, opening of LTCCs may be considered an important basis of multiple cardiac arrhythmias, which may result in sudden death. The present study demonstrated that fast pacing may significantly increase intracellular $\mathrm{Ca}^{2+}$ concentration in atrial myocytes, and verapamil treatment distinctly inhibited this increase, thus suggesting that LTCCs have an important role in $\mathrm{Ca}^{2+}$ influx in the early phase of fast pacing. That verapamil could significantly antagonize rapid pacing-induced increased activation of ERK and p38MAPK indicated that the opening of LTCCs had a close relationship with alterations to the MAPK pathway.
MAPKs are evolutionarily highly conserved protein kinases specific to serine, threonine and tyrosine (20). The MAPK family consists of three major members: p38, ERK and c-Jun N-terminal kinase (JNK). p38MAPK and JNK mediate cell responses to various stressors, and lead to cell death when activated (21). ERK predominantly mediates the proliferation response, with ERK $1 / 2$ as the hub of MAPK signaling pathways (22). In the present study, the expression levels of total ERK1/2, p-ERK1/2 and p-p38MAPK were upregulated $24 \mathrm{~h}$ after fast pacing. MAPK is mainly activated by the $\mathrm{G}$ protein-coupled receptor agonist, and can also be activated by angiotensin II and endothelin-1 via a c-Src dependent mechanism (23). Other factors, such as high osmotic stress, hypoxia/reoxygenation injury, free radicals and reactive oxygen species can also stimulate MAPK activities (24-26). In a previous study, short-term rapid electrical stimulation resulted in a significant upregulation of connexin 43 and 
conduction velocity in cardiomyocytes, via an increased autocrine action of angiotensin II that activated ERK and p38MAPK (27). Pretreatment with losartan, an antagonist of angiotensin type 1 receptor, inhibited vasoconstriction, as well as the activation of ERK1/2 and p38MAPK (28). It may be hypothesized that electric field stimulation results in $\mathrm{Ca}^{2+}$ influx and overload, followed by conformational changes of calmodulin upon $\mathrm{Ca}^{2+}$ binding, and subsequent activation of MAPK signaling molecules by calmodulin binding.

Several nuclear targets for MAPK pathways have been identified, including Elk-1, Ets-1, c-Myc, Sapla, Tal, signal transducer and activator of transcription, c-Jun and activating transcription factor 2 (29). Following mitogen stimulation, MAPK rapidly phosphorylates Elk-1.ERK activation stimulates activator protein (AP)-1 activity via induction of c-Fos, which translocates to the nucleus and combines with pre-existing Jun proteins to form AP-1 dimers that are more stable than those formed by Jun proteins alone (30). In addition, activated ERK1/2 is able to phosphorylate numerous substrates, including various membrane proteins such as CD120a, Syk and calnexin, and cytoplasmic proteins such as MAPK-activated protein kinase and cytosolic phospholipase A2 (31). Activation of the ERK/MAPK signaling cascades may participate in the early structural remodeling of ion channels in AF. The present study demonstrated that inhibition of ERK1/2 and/or p38MAPK was able to rescue the downregulation of $\alpha 1 \mathrm{c}$ and Kv4.3 expression caused by fast pacing. This is necessary and sufficient to demonstrate the above hypothesis. In addition, neither single nor combined use of PD98059 and SB203580 could totally block the fast pacing-induced downregulation of $\alpha 1 \mathrm{c}$ and Kv4.3 expression, thus suggesting the existence of other possible regulatory pathways. In addition, a previous study reported that the function of verapamil to block LTCC did not emerge if fast pacing lasted for over 2 weeks (14); therefore, other factors may participate, such as T-type calcium channels.

In conclusion, the present study demonstrated that the MAPK pathway had an important role in the opening of LTCCs, and interruption of MAPK molecules could affect the expression of ion channels. The reciprocal interaction of the MAPK pathway and ion channels offers therapeutic potential against AF.

\section{Acknowledgements}

The present study was supported by the National Natural Science Foundation of China (grant no. 30600252).

\section{References}

1. Nattel S, Burstein B and Dobrev D: Atrial remodeling and atrial fibrillation: Mechanisms and implications. Circ Arrhythm Electrophysiol 1: 62-73, 2008.

2. Allessie M, Ausma J and Schotten U: Electrical, contractile and structural remodeling during atrial fibrillation. Cardiovasc Res 54: 230-246, 2002.

3. Krogh-Madsen T, Abbott GW and Christini DJ: Effects of electrical and structural remodeling on atrial fibrillation maintenance: A simulation study. PLoS Comput Biol 8: e1002390, 2012.

4. Bosch RF, Scherer CR, Rüb N, Wöhrl S, Steinmeyer K, Haase H, Busch AE, Seipel L and Kühlkamp V: Molecular mechanisms of early electrical remodeling: Transcriptional downregulation of ion channel subunits reduces $\mathrm{I}(\mathrm{Ca}, \mathrm{L})$ and $\mathrm{I}(\mathrm{to})$ in rapid atrial pacing in rabbits. J Am Coll Cardiol 41: 858-869, 2003.
5. Cutler MJ, Jeyaraj D and Rosenbaum DS: Cardiac electrical remodeling in health and disease. Trends Pharmacol Sci 32: 174-180, 2011.

6. Zhao Z, Xie Y, Wen H, Xiao D, Allen C, Fefelova N, Dun W, Boyden PA, Qu Z and Xie LH: Role of the transient outward potassium current in the genesis of early afterdepolarizations in cardiac cells. Cardiovasc Res 95: 308-316, 2012.

7. Voigt N, Li N, Wang Q, Wang W, Trafford AW, Abu-Taha I, Sun Q, Wieland T, Ravens U, Nattel S, et al: Enhanced sarcoplasmic reticulum $\mathrm{Ca}^{2+}$ leak and increased $\mathrm{Na}^{+}-\mathrm{Ca}^{2+}$ exchanger function underlie delayed afterdepolarizations in patients with chronic atrial fibrillation. Circulation 125: 2059-2070, 2012.

8. Kho C, Lee A and Hajjar RJ: Altered sarcoplasmic reticulum calcium cycling - targets for heart failure therapy. Nat Rev Cardiol 9: 717-733, 2012.

9. Parekh AB: Slow feedback inhibition of calcium release-activated calcium current by calcium entry. J Biol Chem 273: 14925-14932, 1998.

10. Millon-Frémillon A, Brunner M, Abed N, Collomb E, Ribba AS, Block MR, Albigès-Rizo C and Bouvard D: Calcium and calmodulin-dependent serine/threonine protein kinase type II (CaMKII)-mediated intramolecular opening of integrin cytoplasmic domain-associated protein-1 (ICAP-1 $\alpha$ ) negatively regulates $\beta 1$ integrins. J Biol Chem 288: 20248-20260, 2013.

11. Wurzinger B, Mair A, Pfister B and Teige M: Cross-talk of calcium-dependent protein kinase and MAP kinase signaling. Plant Signal Behav 6: 8-12, 2011.

12. Goette A, Staack T, Röcken C, Arndt M, Geller JC, Huth C Ansorge S, Klein HU and Lendeckel U: Increased expression of extracellular signal-regulated kinase and angiotensin-converting enzyme in human atria during atrial fibrillation. J Am Coll Cardiol 35: 1669-1677, 2000.

13. Rajadhyaksha A, Husson I, Satpute SS, Küppenbender KD, Ren JQ, Guerriero RM, Standaert DG and Kosofsky BE: L-type $\mathrm{Ca}^{2+}$ channels mediate adaptation of extracellular signal-regulated kinase $1 / 2$ phosphorylation in the ventral tegmental area after chronic amphetamine treatment. J Neurosci 24: 7464-7476, 2004.

14. Goette A, Honeycutt $\mathrm{C}$ and Langberg JJ: Electrical remodeling in atrial fibrillation. Time course and mechanisms. Circulation 94: 2968-2974, 1996

15. Catterall WA: Excitation-contraction coupling in vertebrate skeletal muscle: A tale of two calcium channels. Cell 64: 871-874, 1991.

16. Wehrens XH, Lehnart SE and Marks AR: Intracellular calcium release and cardiac disease. Annu Rev Physiol 67: 69-98, 2005.

17. George AL Jr: Molecular and genetic basis of sudden cardiac death. J Clin Invest 123: 75-83, 2013

18. Priori SG and Chen SR: Inherited dysfunction of sarcoplasmic reticulum $\mathrm{Ca}^{2+}$ handling and arrhythmogenesis. Circ Res 108: 871-883, 2011.

19. Hagenston $A M$ and Bading $H$ : Calcium signaling in synapse-to-nucleus communication. Cold Spring Harb Perspect Biol 3: a004564, 2011.

20. Cargnello M and Roux PP: Activation and function of the MAPKs and their substrates, the MAPK-activated protein kinases. Microbiol Mol Biol Rev 75: 50-83, 2011.

21. Runchel C, Matsuzawa A and Ichijo H: Mitogen-activated protein kinases in mammalian oxidative stress responses. Antioxid Redox Signal 15: 205-218, 2011.

22. Kolch W: Coordinating ERK/MAPK signalling through scaffolds and inhibitors. Nat Rev Mol Cell Biol 6: 827-837, 2005.

23. Yogi A, Callera GE, Montezano AC, Aranha AB, Tostes RC, Schiffrin EL and Touyz RM: Endothelin-1, but not Ang II, activates MAP kinases through c-Src independent Ras-Raf dependent pathways in vascular smooth muscle cells. Arterioscler Thromb Vasc Biol 27: 1960-1967, 2007.

24. Rosette $\mathrm{C}$ and Karin M: Ultraviolet light and osmotic stress: Activation of the JNK cascade through multiple growth factor and cytokine receptors. Science 274: 1194-1197, 1996.

25. Laderoute KR and Webster KA: Hypoxia/reoxygenation stimulates Jun kinase activity through redox signaling in cardiac myocytes. Circ Res 80: 336-344, 1997.

26. Takano H,Zou Y, Hasegawa H, Akazawa H,Nagai T and Komuro I: Oxidative stress-induced signal transduction pathways in cardiac myocytes: Involvement of ROS in heart diseases. Antioxid Redox Signal 5: 789-794, 2003.

27. Nakashima T, Ohkusa T, Okamoto Y, Yoshida M, Lee JK, Mizukami Y and Yano M: Rapid electrical stimulation causes alterations in cardiac intercellular junction proteins of cardiomyocytes. Am J Physiol Heart Circ Physiol 306: H1324-H1333, 2014. 
28. Li Z, Carter JD, Dailey LA and Huang YC: Pollutant particles produce vasoconstriction and enhance MAPK signaling via angiotensin type I receptor. Environ Health Perspect 113: 1009-1014, 2005.

29. Plotnikov A, Zehorai E, Procaccia S and Seger R: The MAPK cascades: Signaling components, nuclear roles and mechanisms of nuclear translocation. Biochim Biophys Acta 1813: 1619-1633, 2011.
30. Karin M: The regulation of AP-1 activity by mitogen-activated protein kinases. J Biol Chem 270: 16483-16486, 1995.

31. Roux PP and Blenis J: ERK and p38 MAPK-activated protein kinases: A family of protein kinases with diverse biological functions. Microbiol Mol Biol Rev 68: 320-344, 2004. 